\title{
Reply to "May Newly Defined Subgenotypes Va and Vb of Newcastle Disease Virus in Poultry Be Considered Two Different Genotypes?"
}

\author{
Leonardo Susta, ${ }^{\text {a }}$ Kiril M. Dimitrov, ${ }^{\mathbf{b}}$ Patti J. Miller, ${ }^{\mathbf{b}}$ Corrie C. Brown, ${ }^{\mathbf{c}}$ Claudio L. Afonso ${ }^{\mathbf{b}}$ \\ Pathobiology Department, University of Guelph, Guelph, Ontario, Canadaa; Southeast Poultry Research Laboratory, USDA, ARS, Athens, Georgia, USA ; Department of \\ Pathology, College of Veterinary Medicine, University of Georgia, Athens, Georgia, USA
}

W e appreciate Dr. Desingu's comments (1) on our paper entitled "Separate Evolution of Virulent Newcastle Disease Viruses from Mexico and Central America” (2). Dr. Desingu points out that the mean evolutionary distance between viruses in subgenotypes $\mathrm{Va}$ and $\mathrm{Vb}$ is higher than the threshold (10\%) set for subgenotype assignment (3) and, therefore, that subgenotypes $\mathrm{Va}$ and $\mathrm{Vb}$ should be considered two distinct genotypes. In addition, he suggests that subgenotype Vc may be considered ancestral to both genotypes $\mathrm{Va}$ and $\mathrm{Vb}$.

In response to the first comment made by Dr. Desingu, we believe that subgenotypes $\mathrm{Va}, \mathrm{Vb}$, and $\mathrm{Vc}$ should remain subgenotypes within genotype $\mathrm{V}$ for the following reasons.

(i) Although we reported an evolutionary distance between subgenotypes $\mathrm{Va}$ and $\mathrm{Vb}(10.9 \%)$ that is slightly higher than the threshold $(2,3)$, the supporting data for a new genotype are not sufficiently strong. In fact, the nucleotide distance criterion $(>10 \%)$ for designating a new genotype should be met for any group of viruses that is compared to all other groups. If subgenotype $\mathrm{Va}$ is to be considered a putative new genotype, it should have an evolutionary distance of $>10 \%$ compared to all other genotypes. Using the same data set as in our previous paper (2), analysis comparing $\mathrm{Va}$ (putative new genotype) to merged $\mathrm{Vb}$ and $\mathrm{Vc}$ (as these will remain and constitute genotype $\mathrm{V}$ ) shows that the distance between them is $9.8 \%$ (Table 1) and thus does not meet the $10 \%$ rule. Similarly, using a new data set with all currently available sequences, the evolutionary distances between these three subgenotypes within genotype $\mathrm{V}$ fall under 10\% (Table 2), confirming our previous results. These observations suggest that above-the-threshold evolutionary distances between subgenotypes do not justify automatic assignment of a new genotype. Calculation of evolutionary distances between subgenotypes is influ-

TABLE 1 Estimates of evolutionary distances between subgenotypes in genotype $\mathrm{V}$, using the full coding sequence of the fusion gene and the previous data set ${ }^{a}$

\begin{tabular}{llll} 
Subgenotype(s) & \multicolumn{2}{l}{ or clade } & \\
\cline { 2 - 4 } or clade & $\mathrm{Va}$ & $\mathrm{Vb}$ & Historical \\
\hline $\mathrm{Va}$ & 0.098 & $(0.008)$ & $(0.007)$ \\
$\mathrm{Vb}+\mathrm{Vc}$ & 0.087 & 0.055 & $(0.004)$ \\
Historical & & \\
\hline
\end{tabular}

${ }^{a}$ The numbers of base substitutions per site resulting from averaging overall sequence pairs between groups within genotype $\mathrm{V}$ are shown. The table includes the same data set $(n=54)$ used in our previous publication (2). Values in parentheses above the diagonal are standard errors, calculated by bootstrap procedure (500 replicates). Analyses were conducted using the maximum composite likelihood model (4), as implemented in MEGA6 (5). The rate variation among sites was modeled with a gamma distribution (shape parameter, 1). The codon positions included were first plus second plus third plus noncoding. All positions containing gaps and missing data were eliminated.
TABLE 2 Estimates of evolutionary distances between subgenotypes in genotype $\mathrm{V}$, using the full coding sequence of the fusion gene and the new data set ${ }^{a}$

\begin{tabular}{llll}
\hline & \multicolumn{2}{l}{$\begin{array}{l}\text { Evolutionary distance for indicated subgenotype } \\
\text { or clade }\end{array}$} \\
\cline { 2 - 4 } $\begin{array}{l}\text { Subgenotype } \\
\text { or clade }\end{array}$ & $\mathrm{Va}$ & $\mathrm{Vb}$ & $\mathrm{Vc}$ \\
\hline $\mathrm{Va}$ & 0.094 & $(0.006)$ & $(0.006)$ \\
$\mathrm{Vb}$ & 0.085 & 0.049 & $(0.003)$ \\
$\mathrm{Vc}$ & & & \\
\hline
\end{tabular}

${ }^{a}$ The numbers of base substitutions per site resulting from averaging overall sequence pairs between groups within genotype $\mathrm{V}$ are shown. The table includes a new data set ( $n=87 ; \mathrm{Va}=42, \mathrm{Vb}=26, \mathrm{Vc}=19)$ of all currently available sequences in genotype $\mathrm{V}$. Values in parentheses above the diagonal are standard errors, calculated by bootstrap procedure (500 replicates). Analyses were conducted using the maximum composite likelihood model (4), as implemented in MEGA6 (5). The rate variation among sites was modeled with a gamma distribution (shape parameter, 1). The codon positions included were first plus second plus third plus noncoding. All positions containing gaps and missing data were eliminated.

enced by how homogeneously such distances are distributed within the genotype and by the number of taxa available for analysis (groups with more strains weigh more heavily in the analysis). Adding or removing few sequences in an analyzed set may affect distances and topology. This is among the reasons explaining why our group has followed a very conservative approach in the creation of new genotypes in the past.

(ii) There are currently no defined criteria for naming genotypes that originate by continued evolution of existing subgenotypes. Under the current classification, a new genotype should receive a different Roman numeral (e.g., genotype XIX) and this will inevitably result in additional confusion among researchers due to lack of consistency with existing literature.

Regarding the second comment of Dr. Desingu stating that subgenotype Vc should be considered ancestral to both subgenotype $\mathrm{Va}$ and subgenotype $\mathrm{Vb}$, we believe that there is no evidence supporting such a statement. As presented in Fig. 1 in our original paper (2), the phylogenetic tree demonstrates that subgenotypes $\mathrm{Vb}$ and $\mathrm{Vc}$ diverged from a common ancestor (bootstrap value, $65 \%$ ) genetically closest to a Newcastle disease virus (NDV) strain

Citation Susta L, Dimitrov KM, Miller PJ, Brown CC, Afonso CL. 2016. Reply to "May newly defined subgenotypes $\mathrm{Va}$ and $\mathrm{Vb}$ of Newcastle disease virus in poultry be considered two different genotypes?" J Clin Microbiol 54:2205-2206. doi:10.1128/JCM.00914-16.

Editor: A. J. McAdam, Boston Children's Hospital

Address correspondence to Leonardo Susta, Isusta@uoguelph.ca.

This is a response to a letter by Desingu et al. (doi:10.1128/JCM.00758-16).

Copyright @ 2016, American Society for Microbiology. All Rights Reserved. 
isolated from a Yellow-Cheeked Parakeet in 1976 in Mexico (KF767469). Similarly, our results show that subgenotype Va diverged independently from a common ancestor of both subgenotypes $\mathrm{Vb}$ and $\mathrm{Vc}$, which is closest to a strain isolated from a BlueFronted Parrot in 1976 in Argentina (KF767470).

We acknowledge the complexities and challenges posed by genotype assignment, and we believe that very stringent and conservative criteria should be used by researchers to properly name taxonomic groups. As with any other RNA virus, Newcastle disease virus is constantly evolving. It is likely that groups of viruses that will eventually meet the classification criteria for consideration as new genotypes will originate from some existing subgenotypes. As a reference, it may be appropriate to utilize rules similar to those set by the WHO/OIE/FAO H5N1 Evolution Working Group for the nomenclature of highly pathogenic H5N1 avian influenza viruses (6). Those rules have been created to split groups into subgroups of higher order (while remaining part of the existing original lower-order group) (e.g., Va.1 or VIg.1 and so on). Indeed, a consortium of international experts has recently been created for the purpose of updating the current nomenclature and for developing more-precise guidelines for defining subgenotypes/genotypes of Newcastle disease virus (Emmanuel Albina, personal communication). We believe that the criteria for assigning of new genotypes and the nomenclature used need to be updated; however, this has to be done based on international consensus rather than by individual scientific teams.

It is our opinion that the ultimate classification of Newcastle disease virus should be made by using complete genome se- quences. The number of available complete genome sequences is increasing progressively, and a vast amount of data are produced by modern sequencers through next-generation sequencing. As shown in Fig. S1 in the supplemental material in our original paper, the complete genome analysis shows that representative strains of all three subgenotypes cluster with very high bootstrap values within genotype $\mathrm{V}(2)$.

\section{REFERENCES}

1. Desingu PA, Dhama K, Malik YS. 2016. May newly defined subgenotypes $\mathrm{Va}$ and $\mathrm{Vb}$ of Newcastle disease virus in poultry be considered two different genotypes? J Clin Microbiol 54:2204. http://dx.doi.org/10 .1128/JCM.00758-16.

2. Susta L, Hamal KR, Miller PJ, Cardenas-Garcia S, Brown CC, Pedersen JC, Gongora V, Afonso CL. 2014. Separate evolution of virulent Newcastle disease viruses from Mexico and Central America. J Clin Microbiol 52: 1382-1390. http://dx.doi.org/10.1128/JCM.00066-14.

3. Diel DG, da Silva LH, Liu H, Wang Z, Miller PJ, Afonso CL. 2012. Genetic diversity of avian paramyxovirus type 1: proposal for a unified nomenclature and classification system of Newcastle disease virus genotypes. Infect Genet Evol 12:1770-1779. http://dx.doi.org/10.1016/j.meegid .2012.07.012.

4. Tamura K, Nei M, Kumar S. 2004. Prospects for inferring very large phylogenies by using the neighbor-joining method. Proc Natl Acad Sci U S A 101:11030-11035. http://dx.doi.org/10.1073/pnas.0404206101.

5. Tamura K, Stecher G, Peterson D, Filipski A, Kumar S. 2013. MEGA6: Molecular Evolutionary Genetics Analysis version 6.0. Mol Biol Evol 30: 2725-2729. http://dx.doi.org/10.1093/molbev/mst197.

6. World Health Organization. 2011. Updated unified nomenclature system for the highly pathogenic H5N1 avian influenza viruses. Last accessed 23 May 2016. http://www.who.int/influenza/gisrs_laboratory/h5 n1_nomenclature/en/. 